\title{
Solvability of discrete Dirichlet problem via lower and upper functions method
}

\author{
IRENA RACHŮNKOVÁ* and LUKÁŠ RACHŮNEK \\ Department of Mathematics, Palacký University, Tomkova 40, 77900 Olomouc, Czech Republic
}

(Received 2 May 2006; revised 27 November 2006; in final form 28 November 2006)

We use the lower and upper functions method to prove the existence of a solution of the Dirichlet problem

$$
\Delta(p(t) \Delta u(t-1))+f(t, u(t))=g(t), \quad t \in[1, T], \quad u(0)=0, \quad u(T+1)=0,
$$

where $T \in \mathbb{N},[1, T]=\{1,2, \ldots, T\}, p:[1, T+1] \rightarrow \mathbb{R}$ is positive and $f:[1, T] \times \mathbb{R} \rightarrow \mathbb{R}$ is continuous. Provided $f$ fulfils certain sign conditions we get the solvability of the problem for each $g:[1, T] \rightarrow \mathbb{R}$.

Keywords: Dirichlet discrete BVP; Lower and upper functions; Brouwer fixed point theorem; Existence

Mathematics Subject Classification 2000: 39A12; 39A10; 39A70

\section{Introduction}

For fixed $T \in \mathbb{N}$ we define the discrete interval $[1, T]=\{1,2, \ldots, T\}$. We will study the Dirichlet problem

$$
\begin{gathered}
\Delta(p(t) \Delta u(t-1))+f(t, u(t))=g(t), \quad t \in[1, T], \\
u(0)=0, \quad u(T+1)=0 .
\end{gathered}
$$

where

$$
\left.\begin{array}{c}
p:[1, T+1] \rightarrow \mathbb{R} \text { is positive, } \quad g:[1, T] \rightarrow \mathbb{R} \\
f:[1, T] \times \mathbb{R} \rightarrow \mathbb{R} \text { is continuous. }
\end{array}\right\}
$$

Here $\Delta$ denotes the forward difference operator with the step size 1, i.e. $\Delta u(t-1)=u(t)-u(t-1)$. Recall that $f(t, x)$ is continuous on $[1, T] \times \mathbb{R}$ if for each $t \in[1, T], f(t, x)$ is a continuous function of $x$.

DeFinITION 1.1. By a solution $u$ of problem (1.1), (1.2) we mean $u:[0, T+1] \rightarrow \mathbb{R}$, $u$ satisfies the difference equation (1.1) on $[1, T]$ and the boundary conditions (1.2).

Discrete boundary value problems arise in the study of solid state physics, chemical reaction, population dynamics and in many other areas, see $[1,13,27]$. Discrete second order nonlinear boundary value problems have been investigated in several monographs (e.g. $[1,5,6,17])$ and

*Corresponding author. Email: rachunko@inf.upol.cz 
papers (e.g. [8-10,14,16,19-21,23,24,26]). Particularly we can refer to papers [2$4,7,11,12,15,18,22,25]$, which deal with various difference equations subjected to Dirichlet conditions. Yongjin Li in Ref. [18] used variational approach and proved the existence of a solution of equations (1.1) and (1.2) under the assumptions

$$
\begin{aligned}
& \exists r>0 \text { such that } x f(t, x) \leq 0 \text { for } t \in[1, T] \text { and }|x| \geq r, \\
& \sum_{t=1}^{T+1}|g(t)|^{2}<\frac{m}{2}, \text { where } m=\min \{p(t): t \in[1, T+1]\} .
\end{aligned}
$$

In this paper, we use a completely different approach based on the lower and upper functions method. By means of this we prove that equation (1.4) yields the solvability of problem (1.1) and (1.2) for each $g[1, T] \rightarrow \mathbb{R}$, i.e. that equation (1.5) can be omitted.

\section{Green's function}

Consider the linear homogeneous equation

$$
\Delta(p(t) \Delta u(t-1))=0, \quad t \in[1, T],
$$

where $p$ satisfies equation (1.3). Define

$$
P(t)=\sum_{i=1}^{t} \frac{1}{p(i)}, \quad t \in[1, T+1] \text { and } P(0)=0,
$$

and denote

$$
M_{p}=\max \left\{\frac{1}{p(t)}: t \in[1, T+1]\right\}>0 .
$$

Remark 2.1. The general solution of equation (2.1) has the form $u(t)=c_{1}+c_{2} P(t)$, $t \in[0, T+1]$, where $c_{1}, c_{2} \in \mathbb{R}$. Therefore equations (1.2) and (1.3) yield $c_{1}=c_{2}=0$ and hence problem (2.1) and (2.2) has only the trivial solution.

Lemma 2.2. Let p satisfy equation (1.3). Then the Green's function of problem (2.1) and (1.2) has the form

$$
G(t, s)=-\left\{\begin{array}{l}
\frac{P(s)}{P(T+1)}(P(T+1)-P(t)) \text { if } 0 \leq s \leq t \leq T+1 \\
\frac{P(t)}{P(T+1)}(P(T+1)-P(s)) \text { if } 0 \leq t \leq s \leq T+1 .
\end{array}\right.
$$

Proof. The proof can be done similarly as in Ref. [17], Example 6.12.

Due to equations (2.2)-(2.4) we see that

$$
\begin{gathered}
G(0, s)=0, \quad G(T+1, s)=0 \text { for } s \in[0, T+1], \\
-T M_{p}<G(t, s)<0 \text { for } t, s \in[1, T] .
\end{gathered}
$$


Further, we have

$$
\Delta G(t-1, s)=\frac{1}{p(t) P(T+1)}\left\{\begin{array}{l}
P(s) \text { for } s+1 \leq t \\
P(s)-P(T+1) \text { for } t \leq s
\end{array}\right.
$$

and

$$
\Delta(p(t) \Delta G(t-1, s))=\left\{\begin{array}{l}
0 \text { for } t \leq s+1 \text { and } t \geq s+1 \\
1 \text { for } t=s .
\end{array}\right.
$$

Therefore, according to Remark 2.1 and Lemma 2.2, we get the following lemma for the nonhomogeneous linear equation

$$
\Delta(p(t) \Delta u(t-1))=g(t), \quad t \in[1, T],
$$

where $p$ and $q$ satisfy equation (1.3).

Lemma 2.3. Problem (2.7) and (1.2) has the unique solution of the form

$$
u_{0}(t)=\sum_{s=1}^{T} G(t, s) g(s), \quad t \in[0, T+1]
$$

\section{Lower and upper functions}

Lower and upper functions are important tools for the investigation of solvability of boundary value problems. Here, we bring their definition for problem (1.1) and (1.2).

Definition 3.1. $\quad \alpha:[0, T+1] \rightarrow \mathbb{R}$ is called a lower function of problem (1.1) and (1.2) if

$$
\begin{gathered}
\Delta(p(t) \Delta \alpha(t-1))+f(t, \alpha(t)) \geq g(t) \text { for } t \in[1, T], \\
\alpha(0) \leq 0, \quad \alpha(T+1) \leq 0 .
\end{gathered}
$$

$\beta:[0, T+1] \rightarrow \mathbb{R}$ is called an upper function of problem (1.1) and (1.2) if

$$
\begin{gathered}
\Delta(p(t) \Delta \beta(t-1))+f(t, \beta(t)) \leq g(t) \text { for } t \in[1, T], \\
\beta(0) \geq 0, \quad \beta(T+1) \geq 0 .
\end{gathered}
$$

THEOREM 3.2 (LOWER AND UPPER FUNCTIONS METHOD). Assume equation (1.3). Let $\alpha$ and $\beta$ be a lower and an upper function of equations (1.1), (1.2) and $\alpha \leq \beta$ on [1,T]. Then problem equations (1.1) and (1.2) has a solution u satisfying

$$
\alpha(t) \leq u(t) \leq \beta(t) \text { for } t \in[0, T+1] .
$$

Theorem 3.2 is a slight modification of Theorem 9.7 in Ref. [17], where $p(t) \equiv 1$. However for the reader's convenience we will prove Theorem 3.2 here. 
Proof. Step 1. For $t \in[1, T], x \in \mathbb{R}$, define function

$$
\tilde{f}(t, x)= \begin{cases}f(t, \beta(t))-\frac{x-\beta(t)}{x-\beta(t)+1} & \text { if } x>\beta(t) \\ f(t, x) & \text { if } \alpha(t) \leq x \leq \beta(t) \\ f\left(t, \alpha(t)+\frac{\alpha(t)-x}{\alpha(t)-x+1}\right. & \text { if } x<\alpha(t) .\end{cases}
$$

Since $\tilde{f}$ is continuous on $[1, T] \times \mathbb{R}$, there exists $M>0$ such that

$$
|\tilde{f}(t, x)| \leq M \text { for } t \in[1, T], \quad x \in \mathbb{R} .
$$

We will study the auxiliary difference equation

$$
\Delta(p(t) \Delta u(t-1))+\tilde{f}(t, u(t))=g(t), \quad t \in[1, T],
$$

and we will prove that problem (3.7) and (1.2) has a solution (see Steps 2 and 3).

Step 2. We define the space

$$
E=\{u:[0, T+1] \rightarrow \mathbb{R}, u(0)=0, u(T+1)=0\}
$$

with the norm $\|u\|=\max \{|u(t)|: t \in[1, T]\}$. Then $E$ is a Banach space with $\operatorname{dim} E=T$. Further, we define an operator $\mathcal{F}: E \rightarrow E$ by:

$$
(\mathcal{F} u)(t)=\sum_{s=1}^{T} G(t, s)(g(s)-\tilde{f}(s, u(s))), \quad t \in[0, T+1] .
$$

Due to equation (1.3), $\mathcal{F}$ is a continuous operator. Denote $B(r)=\{u \in E:\|u\|<r\}$ and

$$
M_{g}=\max \{|g(t)|: t \in[1, T]\} .
$$

Let us choose $r^{*} \geq T^{2} M_{p}\left(M_{g}+M\right)$, where $M_{p}$ and $M$ are given by equations (2.6) and (3.6), respectively. Then by equations (2.5) and (3.8) we get $\mathcal{F}\left(\overline{B\left(r^{*}\right)}\right) \subset \overline{B\left(r^{*}\right)}$. Therefore, the Brouwer fixed point theorem yields the existence of at least one point $u \in \overline{B\left(r^{*}\right)}$ such that $u=\mathcal{F} u$. According to Lemma 2.3 we see that if $u$ is a fixed point of $\mathcal{F}$, then $u$ satisfies equations (3.7) and (1.2).

Step 3. We prove that the solution $u$ of equations (3.7) and (1.2) satisfies equation (1.1). Put $v(t)=\alpha(t)-u(t)$ for $t \in[0, T+1]$ and assume that $\max \{v(t): t \in[0, T+1]\}=v(\ell)>0$. Conditions (1.2) and (3.2) imply $\ell \in[1, T]$. Thus, we have $v(\ell+1) \leq v(\ell)$, $v(\ell-1) \leq v(\ell)$, and consequently $\Delta \alpha(\ell) \leq \Delta u(\ell), \Delta \alpha(\ell-1) \geq \Delta u(\ell-1)$. This leads to $p(\ell+1) \Delta \alpha(\ell) \leq p(\ell+1) \Delta u(\ell), p(\ell) \Delta \alpha(\ell-1) \geq p(\ell) \Delta u(\ell-1)$ and

$$
\Delta(p(\ell) \Delta u(\ell-1)) \geq \Delta(p(\ell) \Delta \alpha(\ell-1)) .
$$

On the other hand, we get by equations (3.1) and (3.7)

$$
\begin{aligned}
\Delta(p(\ell) \Delta \alpha(\ell-1))-\Delta(p(\ell) \Delta u(\ell-1))= & \Delta(p(\ell) \Delta \alpha(\ell-1))-(g(\ell)-\tilde{f}(\ell, u(\ell))) \\
= & \Delta(p(\ell) \Delta \alpha(\ell-1))+f(\ell, \alpha(\ell)) \\
& +\frac{v(\ell)}{v(\ell)+1}-g(\ell) \\
\geq & \frac{v(\ell)}{v(\ell)+1}>0,
\end{aligned}
$$


which contradicts equation (3.10). So, we have proved $\alpha(t) \leq u(t)$ for $t \in[0, T+1]$. The inequality $u(t) \leq \beta(t)$ for $t \in[0, T+1]$ can be proved similarly. Therefore, $u$ satisfies equation (3.5) and hence $u$ is a solution of problems (1.1) and (1.2).

\section{Main results}

Our main result is contained in the next theorem, which provides sufficient conditions for the solvability of problems (1.1) and (1.2). The proof is based on the lower and upper functions method from Theorem 3.2.

THEOREm 4.1. Assume that equations (1.3) and (1.4) hold. Then problem (1.1) and (1.2) has at least one solution.

Proof. By Lemma 2.3, problem (2.7) and (1.2) has the unique solution $u_{0}$ given by equation (2.8). Using equations (3.9), (2.5) and (2.6) we have

$$
\left|u_{0}(t)\right| \leq T^{2} M_{p} M_{g} \text { for } t \in[0, T+1] .
$$

Choose $A, B \in \mathbb{R}$ such that

$$
A \leq-T^{2} M_{p} M_{g}-r, \quad B \geq T^{2} M_{p} M_{g}+r
$$

and define functions

$$
\alpha(t)=u_{0}(t)+A, \quad \beta(t)=u_{0}(t)+B, \quad t \in[0, T+1] .
$$

Then $\alpha(t) \leq-r, \beta(t) \geq r$ for $t \in[0, T+1]$. This implies that $\alpha$ and $\beta$ satisfy equations (3.2) and (3.4), respectively. Moreover, by equation (1.4),

$$
\begin{aligned}
\Delta(p(t) \Delta \alpha(t-1))+f(t, \alpha(t)) & =\Delta\left(p(t) \Delta u_{0}(t-1)\right)+f(t, \alpha(t)) \geq \Delta\left(p(t) \Delta u_{0}(t-1)\right) \\
& =g(t) \text { for } t \in[1, T] .
\end{aligned}
$$

Similarly

$$
\Delta(p(t) \Delta \beta(t-1))+f(t, \beta(t)) \leq g(t) \text { for } t \in[1, T] .
$$

Therefore, $\alpha$ and $\beta$ are a lower and an upper function of equations (1.1) and (1.2), respectively, and $\alpha \leq \beta$ on $[1, T]$. Hence, Theorem 3.2 guarantees the existence of at least one solution $u$ of equations (1.1) and (1.2) satisfying equation (3.5).

Example. Assume $k \in \mathbb{N}, c \in \mathbb{R}, a(t):[1, T] \rightarrow \mathbb{R}, b(t):[1, T] \rightarrow(-\infty, 0)$ and consider the equation

$$
\Delta\left(t^{3} \Delta u(t-1)\right)+a(t)+b(t) u^{2 k-1}(t)=c t^{2} \mathrm{e}^{t}, \quad t \in[1, T] .
$$

By Theorem 4.1, problem (4.1) and (1.2) has a solution. 
COROLlary 4.2. Assume that equation (1.3) holds. Let

$$
\begin{gathered}
g(t)<0, \quad f(t, 0) \geq 0 \text { for } t \in[1, T], \\
\exists r>0 \text { such that } f(t, x) \leq 0 \text { for } t \in[1, T] \text { and } x \geq r .
\end{gathered}
$$

Then problem (1.1) and (1.2) has a solution u such that

$$
u(t)>0 \text { for } t \in[1, T] \text {. }
$$

Proof. Condition (4.2) implies that if we put $\alpha(t) \equiv 0$, we have

$$
\Delta(p(t) \Delta \alpha(t-1))+f(t, \alpha(t))=f(t, 0) \geq 0>g(t), \quad t \in[1, T] .
$$

If $\beta(t)$ is the same as in the proof of Theorem 4.1, we get a solution $u$ of problem (1.1), (1.2) such that

$$
0 \leq u(t) \leq \beta(t) \text { for } t \in[0, T+1] .
$$

Since $f(t, 0)>g(t)$ on $[1, T]$, we obtain equation (4.4).

COROLlary 4.3. Assume that equation (1.3) holds. Let

$$
\begin{gathered}
g(t)>0, \quad f(t, 0) \leq 0 \text { for } t \in[1, T], \\
\exists r>0 \text { such that } f(t, x) \geq 0 \text { for } t \in[1, T] \text { and } x \leq-r .
\end{gathered}
$$

Then problem (1.1) and (1.2) has a solution u such that:

$$
u(t)<0 \text { for } t \in[1, T]
$$

Proof. We argue similarly as in the proof of Corollary 4.2 putting $\alpha(t)$ as in the proof of Theorem 4.1 and $\beta(t) \equiv 0$.

Example. If $a(t) \geq 0$ on $[1, T]$ and $c<0$, then by Corollary 4.2, problem (4.1) and (1.2) has a solution, which is positive on $[1, T]$.

If $a(t) \leq 0$ on $[1, T]$ and $c>0$, then by Corollary 4.3, problem (4.1) and (1.2) has a solution, which is negative on $[1, T]$.

\section{Acknowledgements}

Supported by the Council of Czech Government MSM 6198959214.

\section{References}

[1] Agarwal, R.P., 2000, Difference Equations and Inequalities. Theory, Methods and Applications, Revised and expanded 2nd ed. (New York: Marcel Dekker). 
[2] Agarwal, R.P. and O'Regan, D., 1999, Singular discrete boundary value problems. Applied Mathematics Letters, 12, 127-131.

[3] Agarwal, R.P. and O'Regan, D., 1998, Difference equations in abstract spaces. Journal of the Australian Mathematical Society (Series A), 64, 277-284.

[4] Agarwal, R.P. and O'Regan, D., 2000, Nonpositone discrete boundary value problems. Nonlinear Analysis, 39, $207-215$.

[5] Agarwal, R.P., O'Regan, D. and Wong, P.J.Y., 1999, Positive Solutions of Differential, Difference and Integral Equations (Dordrecht: Kluwer).

[6] Agarwal, R.P. and Wong, P.J.Y., 1997, Advanced Topics in Difference Equations (Dordrecht: Kluwer).

[7] Anderson, N. and Arthurs, A.M., 1985, A class of second-order nonlinear difference equations. I: Extremum principles and approximation of solutions. Journal of Mathematical Analysis and Applications, 110, 212-221.

[8] Atici, F.M., Cabada, A. and Otero-Espinar, V., 2003, Criteria for existence and nonexistence of positive solutions to a discrete periodic boundary value problem. Journal of Differential Equations and Applications, $\mathbf{9}$ $765-775$.

[9] Atici, F.M. and Guseinov, G.SH, 1999, Positive periodic solutions for nonlinear difference equations with periodic coefficients. Journal of Mathematical Analysis and Applications, 232, 166-182.

[10] Avery, R.I., 1998, Three positive solutions of a discrete second order conjugate problem. PanAmerican Mathematical Journal, 8, 79-96.

[11] Bereanu, C. and Mawhin, J., Existence and multiplicity results for nonlinear second order difference equations with Dirichlet boundary conditions. Mathematica Bohemica, to appear.

[12] Cabada, A. and Otero-Espinar, V., 2002, Existence and comparison results for difference $\phi$-Laplacian boundary value problems with lower and upper solutions in reverse order. Journal of Mathematical Analysis and Applications, 267, 501-521.

[13] Cahn, J.W., Chow, S.N. and Van Vleck, E.S., 1995, Spatially discrete nonlinear diffusion equations. Rocky Mountain Journal of Mathematical, 25, 87-118.

[14] Dannan, F., Elaydi, S. and Liu, P., 2000, Periodic solutions to difference equations. Journal of Differential Equations and Applications, 6, 203-232.

[15] He, Z., 2003, On the existence of positive solutions of $p$-Laplacian difference equations. Journal of Computational and Applied Mathematics, 161, 193-201.

[16] Henderson, J. and Thompson, H.B., 2001, Difference equations associated with fully nonlinear boundary value problems for second order ordinary differential equations. Journal of Differential Equations and Applications, 7, 297-321.

[17] Kelley, W.G. and Peterson, A.C., 2001, Difference Equations. An Introduction with Applications, 2nd ed. (San Diego: Academic Press).

[18] Li, Y., 2006, The existence of solutions for second-order difference equations. Journal of Differential Equations and Applications, 12, 209-212.

[19] Ma, R. and Reffoul, Y.N., 2004, Positive solutions of three-point nonlinear discrete second order boundary value problem. Journal of Differential Equations and Applications, 10, 129-138.

[20] Mawhin, J., Thompson, H.B. and Tonkes, E., 2004, Uniqueness for boundary value problems for second order finite difference equations. Journal of Differential Equations and Applications, 10, 749-757.

[21] Rachůnková, I. and Rachůnek, L., Singular discrete second order BVPs with $p$-Laplacian. Journal of Differential Equations and Applications, to appear.

[22] Rachůnková, I. and Tisdell, C., Existence of non-spurious solutions to discrete boundary value problems. Australian Journal of Mathematical Analysis and Applications, to appear.

[23] Thompson, H.B. and Tisdell, C., 2002, Boundary value problems for systems of difference equations associated with systems of second-order ordinary differential equations. Applied Mathematics Letters, 15, 761-766.

[24] Thompson, H.B. and Tisdell, C., 2003, The nonexistence of spurious solutions to discrete, two-point boundary value problems. Applied Mathematics Letters, 16, 79-84.

[25] Wang, Y.-M., 1998, Monotone methods for a boundary value problem of second-order discrete equation. Computers and Mathematics with Applications, 36, 77-92.

[26] Zhang, L. and Jiang, D., 2003, Monotone method for second order periodic boundary value problems and periodic solutions of delay difference equations. Applied Analysis, 82, 215-229.

[27] Zimmer, B., 1991, Stability of travelling wavefronts for the discrete Nagumo equation. SIAM Journal of Mathematical Analysis, 22, 1016-1020. 